\title{
THE HERTZSPRUNG-RUSSELL DIAGRAMS OF OLD STELLAR POPULATIONS
}

\author{
W. A. BAUM \\ Mount Wilson and Palomar Observatories
}

\section{INTRODUCTION}

The color-magnitude diagrams of globular star clusters are described and attention is called to some evolutionary implications. The predominant stellar population of large elliptical galaxies is shown to be unlike that of globular star clusters but probably similar to that of the older stars in the disk of our Galaxy. The same is true of the population in the nuclei of spiral galaxies. Dwarf ellipticals, on the other hand, are found to have a globular-cluster-type population. The kind of stellar population predominant in a galaxy seems to depend upon the magnitude and the rotational flattening of the galaxy. A unified evolutionary hypothesis is proposed to account for the existence of galaxies of different types.

HR D I A G R A M S

The oldest stars in our Galaxy are evidently those in the halo; they still retain the very high random velocities that must have existed in the pre-stellar material during a very early era. From an evolutionary point of view, it will therefore be appropriate to open the discussion with some comments on the observed features in the HR diagrams of halo inhabitants, i.e., globular clusters. We can then extend the discussion to old populations in general.

The form of the HR diagram for a globular cluster is well known. The diagram for Messier I3, shown in Figure I, will serve as a suitable example. The circles [I] and crosses [2] all represent photoelectric observations. The branch of the diagram below Igth magnitude is the main sequence. Joining it at rgth magnitude is the subgiant-giant branch which reaches upward at the right to rath magnitude. Extending leftward from the red giant branch is the so-called horizontal branch; the zone of the cluster-type variables is indicated by the rectangle at $5^{\text {th }}$ magnitude.

Other globular clusters all seem to have HR diagrams of the same general form but with observable differences in the exact positions and shapes of the branches [3]. A sample comparison of Mr3 with $\mathrm{M}_{3}$ and $\mathrm{M}_{92}$ is shown in Figure 2. Differences in the shape and tilt of the red-giant branch and in the form of the horizontal branch are well established. There are also appreciable differences in the relative populousness of the horizontal branch, particularly on the redward side of the variable-star zone. Some of these differences appear to be correlated with one another and with the number of cluster-type variables; consequently, the number of variables probably provides a meaningful index for classifying globular clusters [4]. It should be mentioned, however, that the spectroscopic appearance of the red-giant branch and its lateral position in the HR plane do not seem to be well correlated with the other characteristics, and might accordingly be regarded as providing a second index of cluster type. 
Using the number of cluster-type variables as a classification index, we find that the variable-poor clusters lie preferentially closer to the galactic plane than the other clusters [I]. This suggests that the variable-poor clusters may have been formed during a slightly later stage in the dynamic relaxation of the Galaxy. Since the relative populousness of the horizontal branch is among the characteristics correlated with the variable-star index, we should expect the clusters near the galactic plane to have later integrated spectral types than the others. This is exactly what Morgan [5] has found.

The relative positions of the main sequences in the HR diagrams of various globular clusters are not easy to establish with precision, partly because the main sequences are observationally difficult to reach and partly because the relative adjustment of the absolute-magnitude

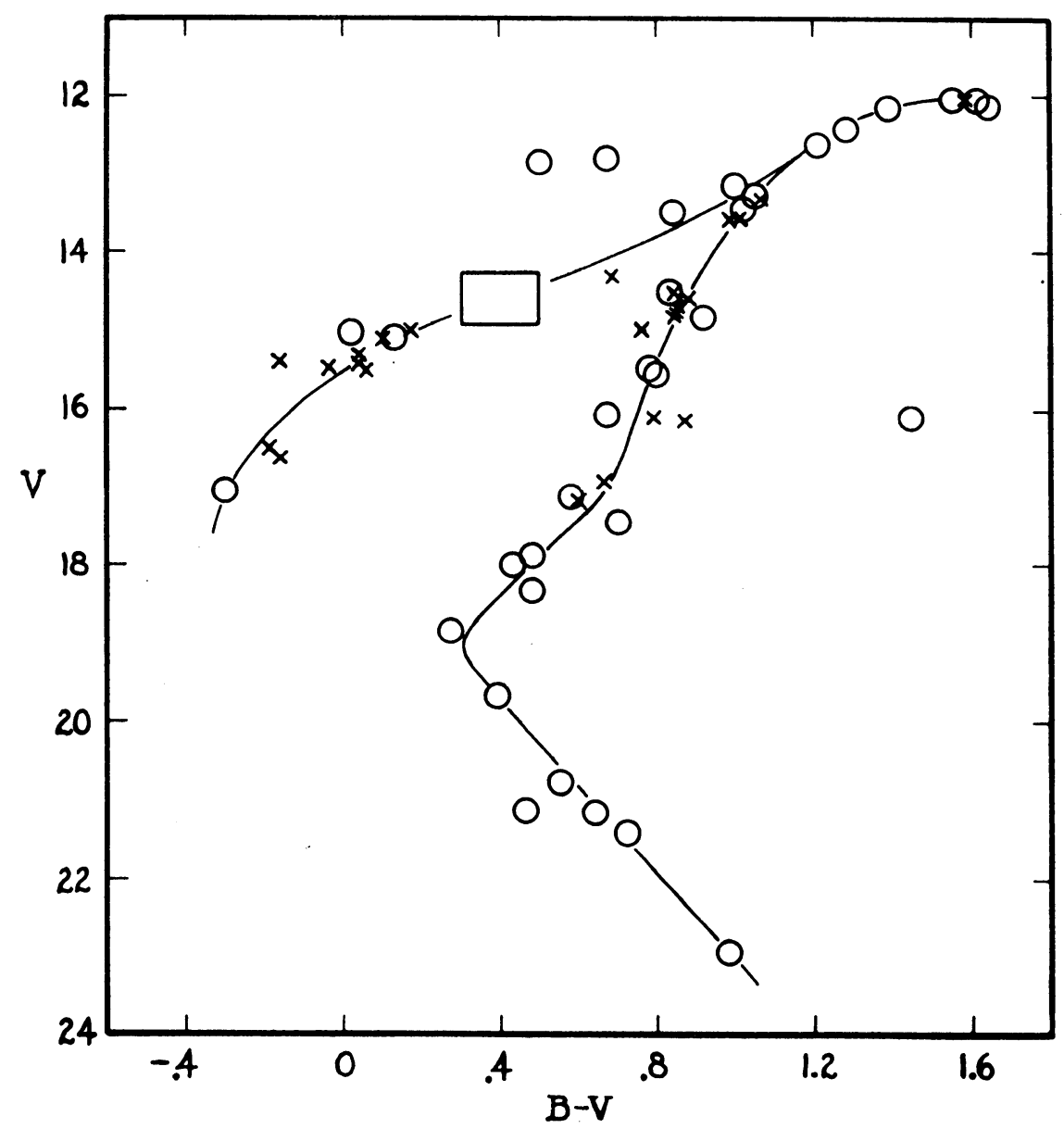

FIGURE I. Photoelectric HR diagram of Messier I3. O = A. J. 59, 425, 1954. $x=$ Ap. J. 122. 172,1955 . New work on the main sequence is now in progress.

scale in each cluster is subject to assumptions. From an evolutionary point of view, one can argue that the absolute bolometric magnitude at which the sub-giant branch now departs from the main sequence should be approximately the same for all globular-clusters if, as is usually supposed, they are of comparable ages. The locus of the departure is represented by the dotted line in Figure 2. If the cluster-type variables in $M_{3}$ are adjusted to $M_{v} \sim 0$, the main 
sequence [6] falls quite close to an evolved main sequence for the solar neighborhood. The main sequence of $\mathrm{Mr}_{3}$, on the other hand, falls at the left in the sub-dwarf region.. If the modulus relationship in Figure 2 is assumed and if no corrections for interstellar reddening are introduced, the photoelectric data of 1953 for the Mr3 main sequence [ $\mathrm{I}$ ] yield an excess blueness of 0.2 magnitude relative to $\mathrm{M}_{3}$ with an uncertainty of about half that amount. New data

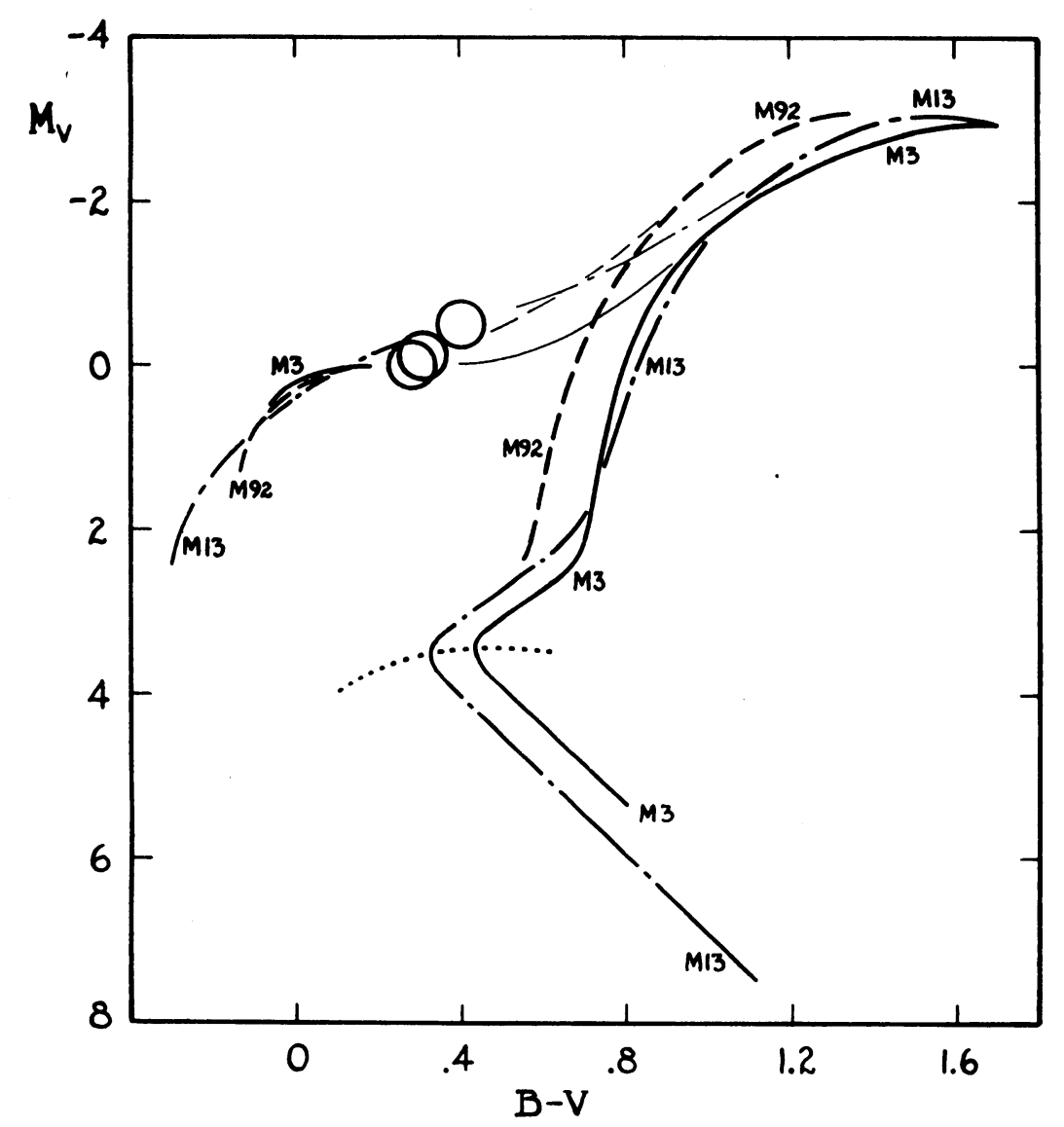

FIgURE 2. A comparison of HR diagrams for $\mathrm{Mr}_{3}, \mathrm{M}_{3}$ and $\mathrm{M}_{92}$.

obtained this spring under a joint program [7] have established the position of the Mr3 main sequence more accurately, the present estimate of the excess blueness being nearer o.I magnitude.

If, however, the cluster-type variables in $\mathrm{M}_{3} 3$ are lowered to the conventional value of $M_{v} \sim 0$ and if reddening corrections are applied, the excess blueness of $\mathrm{Mr}_{3}$ becomes roughly 0.25 magnitude. Although differences in the blanketing effect of spectral lines must be very large in order to explain a large blue excess, it is necessary to assume the reality of such an excess in MI3 if the ages usually ascribed to globular clusters are to be consistent with SchönbergChandrasekhar [8] evolution. In short, the initial suggestion [I] of placing the main sequence of $\mathrm{MI}_{3}$ in the sub-dwarf region still appears preferable to elevating the cluster-type variables much above $M_{v} \sim 0$. The accuracy of observations in other clusters such as $M_{3}$ and $M_{92}$ must be improved before more can be said about their relationships in this regard.

Any successful theory of stellar evolution must be able to provide a reasonable explanation for all features of all observed HR diagrams, including those of globular clusters. Two 
well known theories have been proposed. According to one theory, stars remain in the vicinity of the main sequence until ro per cent of their hydrogen has been consumed [8]. It is assumed that there is negligible internal mixing and no significant loss of mass. They then evolve into the giant phase and consume their remaining fuel more rapidly [9]. According to the other theory [10], stars evolve downward along the main sequence, losing mass as they go; they are assumed to be mixed, and the rate of mass loss is so chosen as to preserve a dispersionless massluminosity relation. The HR diagrams for globular clusters offer some important clues bearing on the choice between these two theories.

In the first place, the branches are narrow and contiguous all the way from the main sequence up through the giant branch and back across the horizontal branch. There are only three alternative explanations for the presence of the branches above the main sequence: either $[\mathrm{I}]$ the stars belonging to these upper branches have evolved away from the main sequence, or [2] they are evolving toward the main sequence, or [3] their individual evolutionary tracks do not join the main sequence. The first of these possibilities is represented by the theory outlined in references 8 and 9, and it seems to provide a reasonable basis for understanding the observed HR diagrams. The second possibility, if considered in terms of gravitational contraction, would require the recent manufacture of new stars in globular clusters; that would be unthinkable. The third possibility also appears unlikely, because it requires the invention of more than one fundamental stellar model to explain why some stars take up residence on the main sequence while others in the same cluster do not.

It should also be noted that the blue end of the horizontal branch joins neither the main sequence nor an upward extrapolation of the main sequence; it crosses the main-sequence region and fades out at extremely blue color indices. The horizontal branch is easily accountable as the final segment of a continuously traceable evolutionary route followed by stars which have evolved away from the main sequence according to references 8 and 9 , but it would not be easily understood as an avenue of injection for stars being fed into the main sequence as might be inferred from reference ro.

Two additional clues deserve mention in connection with globular cluster diagrams. Sandage $[\mathrm{II}]$ has called attention to the star $\zeta$ Herculis A, which has an accurately determined mass in good agreement with the value that would be predicted on the assumption that it has evolved away from the main sequence along a track similar to those computed empirically for the sub-giants in globular clusters. Von Hoerner [12], using star counts by Sandage [I3] and by Tayler [I4], has found that the radial distribution of the stars in a globular cluster implies a mass-luminosity relation which is consistent with the belief that the brighter stars have evolved from a relatively small segment of the main sequence and that they have masses smaller than those predicted by the ordinary mass-luminosity relation.

In short, it seems difficult to account for the upper branches of globular cluster HR diagrams except by supposing that stars evolve away from the main sequence instead of along it.

Note added in proof : A letter recently received from Dr. A. G. Massevitch indicates that evolution with decreasing mass, as set forth in reference Io, is proposed only for massive stars $(\mathscr{M}>2 \odot$ ) of Population I. Dr. Massevitch believes that the observational data for stars more massive than $5 \odot$ are in accord with reference Io, but she agrees that globular cluster HR diagrams are best explained by the evolution of stars $(\mathscr{M} \sim \mathrm{I} \odot)$ away from the main sequence. In other words, she feels that both kinds of evolutionary behavior exist and that the problem is to determine the circumstances conducive to each. 


\section{STELLAR POPULATIONS IN GALAXIES}

It have been tempting to assume that all old populations should have HR diagrams like those of globular clusters. Data now available clearly indicate that this is not true. As we have seen, globular clusters even differ appreciably among themselves. There is no fundamental reason that old populations should all have the same chemical abundances and follow the same evolutionary tracks. It is of interest here to summarize the evidence for major differences among old stellar populations and to propose a general evolutionary model for the stellar content of galaxies.

Let us suppose a very old system of stars with approximately the same chemical abundances as the majority of stars in the solar neighborhood. What would the HR diagram look like? The answer is evident from an intercomparison of the HR diagrams for various galactic clusters [I5]. Consistent with the evolutionary theory favored by the arguments above [8], [9], the prime example of an old galactic cluster is Messier 67. It has a main sequence below $\mathrm{M}_{\mathrm{v}} \sim+3$ and it has stars in the normal giant region, but it has no members above $\mathrm{M}_{\mathrm{v}} \sim \mathrm{o}$ and it has no apparent horizontal branch. This last point is important because a population based on this model would exhibit practically no characteristics attributable to stars earlier than F5. Let us, then, regard M67 as an example of what we mean by "old disk population".

To imagine that all stellar populations can be cleanly separated into two distinct homogeneous types would be an oversimplification [I6]. As a matter of practical convenience, however, we can make an analysis in terms of two extreme models occurring in mixed proportions. Both of these models can be based directly on observed prototypes. One of them can be the old disk population whose HR diagram is described above; the other can be called "halo population" and can be characterized by an HR diagram of the globular-cluster type. The

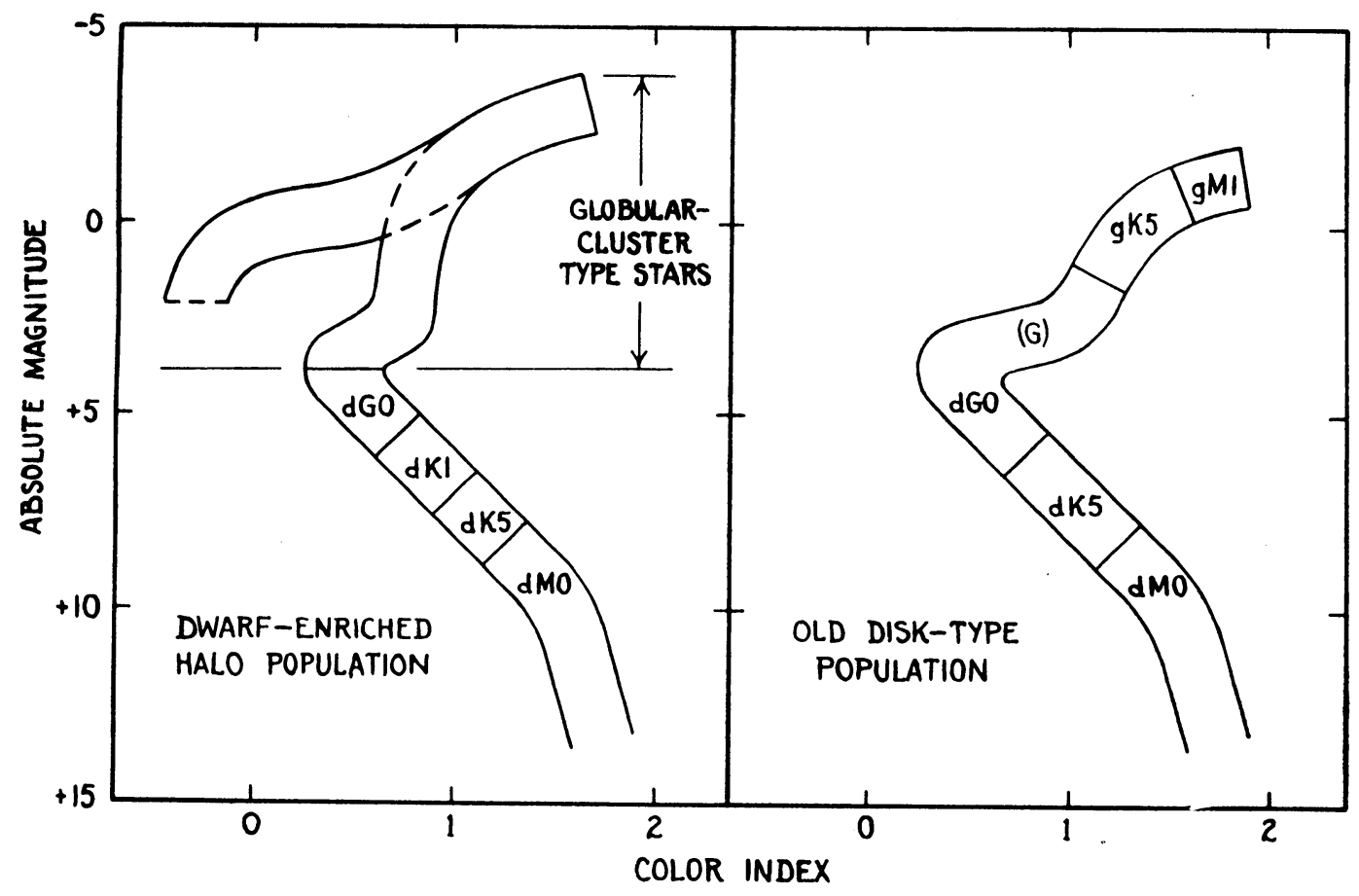

FIGURE 3. Schematic HR diagrams of two models for old populations (Sky and Telescope 14, 373, 1955). The segments represent ingredients adopted for computational purposes. 
two models [I7] are compared schematically in Figure 3, in which the HR diagrams are divided into segments whose relative contributions we shall examine.

The two models differ mainly in the presence (or at least in the importance) of the horizontal branch and in the color-magnitude position of the giant branch. These differences are supposedly manifestations of a difference in metal abundance. If metals have been produced in the course of time by nucleogenesis [32] in a relatively few fast-living stars of very great mass and have been discharged into interstellar space when those stars exhausted themselves, we might expect that stars of moderate mass should have metal abundances related to their times and places of formation. The very oldest stars (halo population) should accordingly have had the least oppotunity to acquire a supply of ready-made metals.

The purpose of formulating the two models in Figure 3 is to provide a means by which the stellar content of external galaxies can be inferred from integrated properties. With the exception of the Magellanic Clouds and a few very tenuous dwarf systems close enough for individual stars to be observed down to a limit below $\mathrm{M}_{\mathrm{v}}=0$, we must derive most of our information regarding the stellar content of galaxies from their integrated light. The properties of principal interest will be the count-brightness ratio, the spectral-energy distribution, the massluminosity ratio, and spectroscopic features.

The count-brightness ratio is obtained by counting the number of photographically resolved stars and dividing the count by the surface brightness. Figure 4 shows the luminosity functions for the two models, the halo-population model being treated at first without dwarf enrichment. The curves have been normalized to equal integrated brightness. If, for example, we can resolve and count all stars brighter than $\mathrm{M}_{\mathrm{v}}=-2$, we should expect to obtain a much higher count for a halo-type population than for an old disk-type population of the same integrated surface brightness.

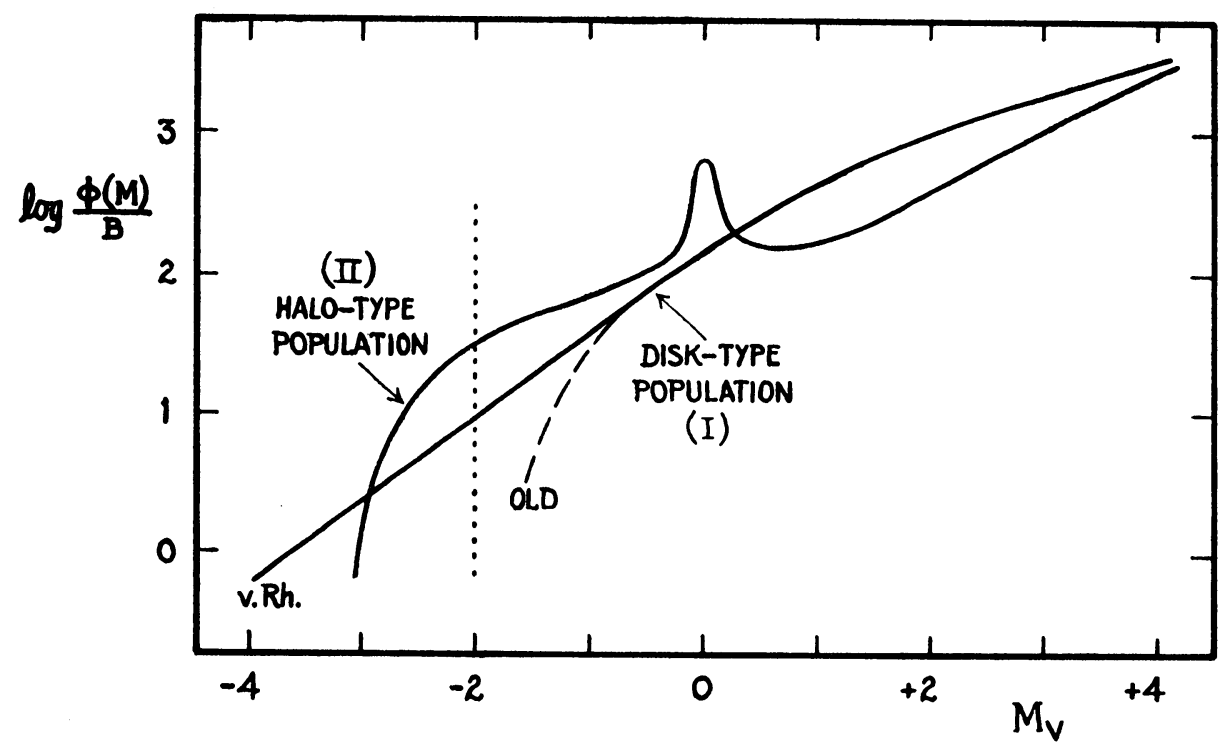

Figure. 4 Luminosity functions for two populations of equal integrated brightness (A. J. 60, 248, 1955).

The count-brightness ratio was applied several years ago [18] to the Andromeda galaxy, $\mathrm{M}_{31}$, and to its "blue" elliptical companion, NGC 205. It was found that the count-brightness ratio of NGC 205 agreed with that of a non-enriched halo-population model but that the main 
body of M3I, exclusive of spiral arms, might best be described as predominantly an old disk population plus a minor contribution from a halo-type population. This conclusion concerning M3I was consistent with Schwarzchild's interpretation [19] of the mass-luminosity ratio.

It was also possible to apply [I7] the same line of reasoning to the nucleus region of M3I simply by noting the minimum radius (3.5) at which resolution was initially reported by Baade [20]; on the basis of the surface brightness at that radius, a pure halo-population model would predict a density of threshold images which could not have been photographically resolved. The evidence therefore favored an old-disk-population model for the nucleus of M3I as well as for the disk itself. This conclusion was consistent with the fact that the nuclear color of $\mathrm{M}_{3} \mathrm{I}$ is similar to that of $\mathbf{M}_{32}$, whose stellar content is discussed below. It has also been confirmed by the recent spectroscopic analysis of Morgan and Mayall [2I].

The stellar content of M32 was first examined [I7] by means of six-color data already in the literature [22]. Photometric data for the ingredients comprising each model in Figure 3 were added together in various proportions to determine which model could be adjusted to yield the better six-color fit to the data for $\mathrm{M}_{32}$. The dwarf content of the halo-population model was necessarily allowed to differ from that of a globular cluster, but the distribution of stars along the upper branches was assumed to be pre-determined by evolution and therefore not divisible into independent segments. The best compromise fit to the $\mathrm{M}_{32}$ data was obtained with an old-disk-population model having about the same proportions of ingredients as the prototype, M67; specifically, about one-third of the visual light could be ascribed to giants, and about two-thirds to sub-giants and stars on the main sequence. A specific argument against the halo-population model was its inability to provide the right amount of ultraviolet light. It should be emphasized, however, that a minority representation of a halo-type population, as suggested by the resolved red giants around $\mathrm{M}_{\mathrm{v}} \sim-3$, would not be at variance with these conclusions.

The same six-color data for $\mathrm{M}_{32}$ have also been examined by Roberts [23] in terms of the halo-population model, but no comparison with an alternative model was made.

Quite a few elliptical galaxies with widely differing redshifts have been observed photoelectrically at Palomar in six to eight colors, some by Whitford [24] and some by the writer [25]. The results indicate that large ellipticals are all basically similar to one another in stellar content

Table I

Synthetic elliptical Galaxy fitted in eight colors

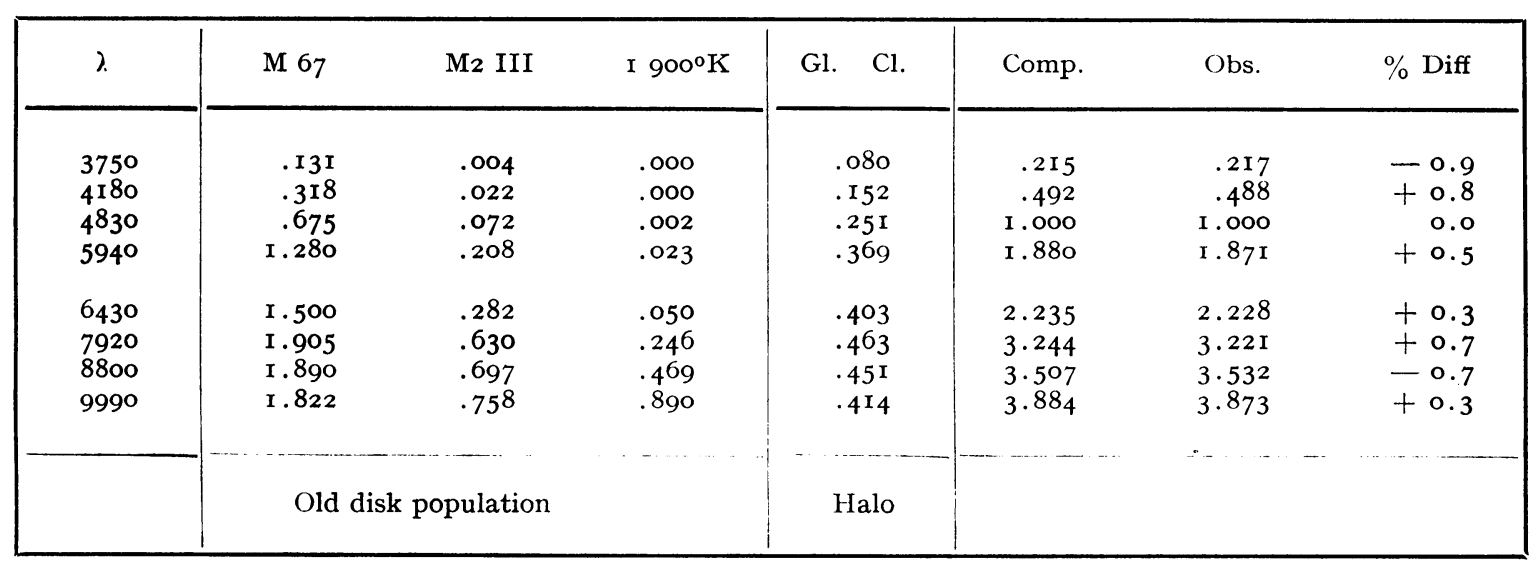


and that they differ only a little from M32. Two large nearby ellipticals were measured recently in eight colors by Tifft [26] at Mount Wilson and were analyzed in terms of population models. When Tifft's data are re-examined in terms of the models being compared here, an excellent fit is obtained with the combination of ingredients shown in Table I. At visual wavelengths, the halo-type population contributes about 20 per cent of the integrated light, but bolometrically it accounts for less than 5 per cent; the rest is contributed by old-disk-type population. The combined model fits within less than I per cent at all wavelengths from $3750 \AA$ to nearly I $\mu$.

In order to represent old-disk-type population more exactly, the M67 prototype has been supplemented in Table I by $M$ giants plus a still cooler component. As pointed out in an earlier paper [27], additions of that kind are required by evolutionary considerations. This refinement is appropriate here because of the greater information which the eight-color system provides in the infrared region.

An interesting observation has been made recently by Code [28] with a photoelectric spectrum scanner. Ellipticals and spectroscopically similar systems (such as the nucleus of M3I) are found to have a general depression due to a blend of lines around $\lambda 5400$ of the same strength found in giant stars. This suggests that most of the K-type light is contributed by

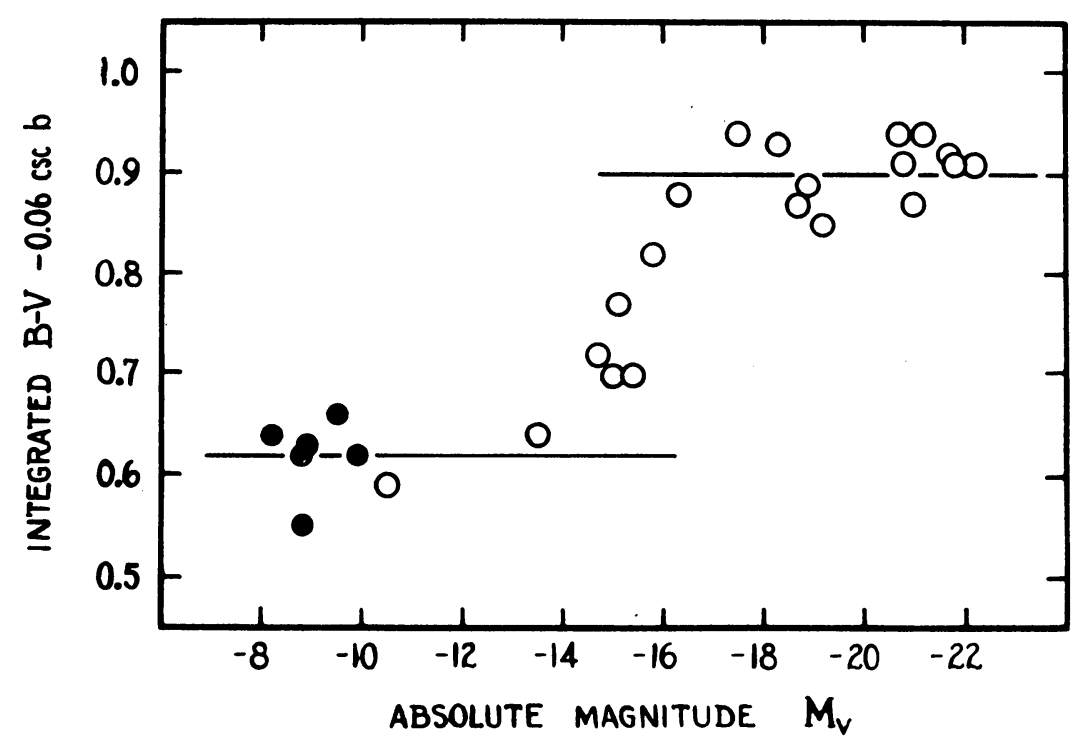

FIGURE 5. Intrinsic color indices of old stellar systems. = globular clusters, $\mathrm{O}=$ elliptical galaxies.

giants. Code's observation therefore provides additional evidence in favor of old-disk-type population for the predominant constituent of ellipticals.

Mass-luminosity ratios inferred from these models are of limited value in providing any test, because the masses depend too critically upon the absolutely faint stars about which the integrated light tells us very little, and because we are not yet prepared to say exactly what role is played by non-luminous matter.

To summarize : By making certain reasonable assumptions, one can infer the general forms of the HR diagrams for old stellar systems by investigating their integrated properties. It has been concluded that stars similar to the older members of the disk population in the solar neighborhood are the predominant type in the disk of $\mathrm{M}_{3} \mathrm{I}$ (excepting its spiral arms), 
in $\mathrm{M}_{32}$, and in various large ellipticals. Conversely, the less massive elliptical NGC 205 is thought to have an HR diagram similar to that of a globular cluster.

Now, what do we know about the very tenuous dwarf galaxies such as the Draco System, in which a globular-cluster-type HR diagram has been directly observed by Baade and Swope [29]? The integrated color indices of dwarf galaxies originally estimated photographically by Holmberg [30] were puzzlingly similar to those of large ellipticals, but recent photoelectric observations [27] yield integrated color indices similar to those of globular clusters.

The color indices of old systems of all sizes, ranging from globular clusters to large elliptical galaxies, are plotted in Figure 5 as a function of absolute magnitude. The obvious inference, of course, is that old systems fainter than $M_{v} \sim-I_{5}$ are composed mainly of stars

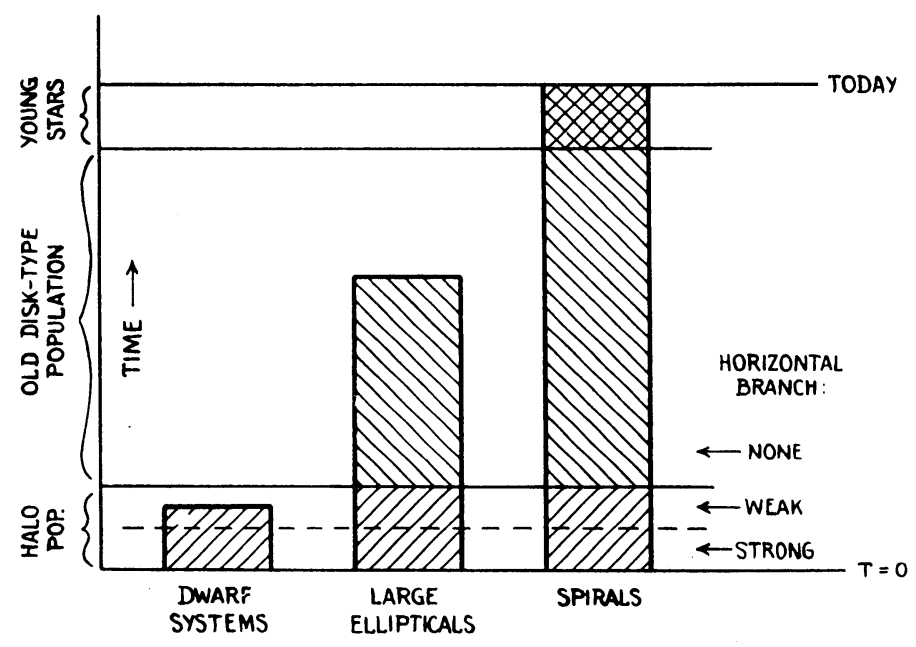

FIGURE 6. Galaxies distinguished by the duration of star formation.

fitting a globular-cluster-type (halo population) HR diagram, whereas old systems brighter than $\mathrm{M}_{\mathrm{v}} \sim$ - I5 are composed mainly of stars fitting an HR diagram of the old-disk-population kind.

How might this difference have come about physically? In our own Galaxy the halopopulation stars have very high random velocities and presumably were formed during a very brief era at the very beginning of the history of the Galaxy. The duration of this initial turbulent era has been estimated by Schwarzschild [3I] to have been less than a half billion years. Recalling that certain characteristics of globular clusters are correlated with their locations in the Galaxy [4], we conclude that the outer clusters now characterized by strong horizontal branches must have been formed first, that the inner clusters with weak horizontal branches were formed later, and that the disk clusters with practically no horizontal branches were formed last.

I should therefore like to propose the evolutionary hypothesis illustrated schematically in Figure 6. In a dwarf system we suppose that star formation has not progressed beyond an initial halo-population stage, either because the duration of that stage is longer in a dwarf system or because star formation ceased early. In the larger ellipticals we suppose that star formation continued considerably past the halo-population stage but came to a halt before the present day. To complete this picture, we suppose that moderately large systems with high angular momenta and a high degree of flattening are the only ones in which star formation 
has not yet stopped; such systems are, of course, the spirals. Thus, the evolutionary history of a galaxy is suggested to be largely predetermined by two initial conditions : one is its mass, and the other is its angular momentum.

Evidently these two conditions influence the duration of star formation and the distribution of heavy elements created by nucleogenesis [32]. Very likely the density of interstellar matter and the strengths of interstellar magnetic fields are factors involved. We do not need to appeal to primordial differences in chemical abundances.

\section{REFERENCES}

[I] W. A. Baum, A. J. 59, 422, 1954.

[2] H. Arp and H. Johnson, Ap. J. 122, I 7 I, 1955.

[3] H. Arp, W. Baum, and A. Sandage, A. J. 57, 4, 1952.

W. A. Baum, A. J. 57, 222, 1952.

H. C. Arp, A. J. 60, 3I 7, 1955.

A. R. Sandage, Mem. Soc. Roy. Sci. Liège, Ser. IV, 14, 254, 1953.

W. A. Baum, A. J. 59, 429, I954.

[4] H. C. Arp, A. J. 60, 334, 1955.

W. A. Baum, Smithsonian Contr. to Ap. 1, $165,1956$.

[5] W. W. Morgan, P. A. S. P. 68, 5 I I, 1956.

[6] H. L. Johnson and A. R. Sandage, Ap. J. 124, 387, 1956.

[7] W. A. Hiltner, H. L. Johnson, A. R. Sandage, and W. A. Baum.

[8] M. Schönberg and S. Chandrasekhar, AP. J. 96, I6I, I942.

[9] J. Oke and M. Schwarzschild, Ap. J. 116, 317, 1952.

A. Sandage and M. Schwarzschild, Ap. J. 116, 463, 1952.

A. E. Roy, M. N. 112, 484, 1952 .

A. R. Sandage, Mem. Soc. Roy. Sci. Liège, Ser. IV, 14, 254, 1954.

[10] V. A. Ambartsumian, Trans. I. A. U. 8, 673, 1952.

V. G. Fesenkov, Trans. I. A. U. 8, 707, 1952.

A. G. Massevitch, Mem. Soc. Roy. Sci. Liege, Ser. IV, 14, 267, 1954.

A. G. Massevitch, this volume p. 89 .

[II] A. R. Sandage, Mem. Soc. Roy. Sci. Liège, Ser. IV, 14, 267, I954.

[I2] S. von Hoerner, Ap. J. 125, 45I, 1957.

[13] A. R. Sandage, A. J. 59, 162, 1954.

[I4] R. J. Tayler, A. J. 59, 4I3, I 954.

[15] H. Johnson and A. Sandage, Ap. J. 121, 623, I955.

[16] J. H. Oort, Ricerche Astronomiche 5, 4I5, 1958. Proceedings of the Conference on Stellar Populations, edited by D. J. K. O'Connell, S. J., Specola Vaticana.

[17] W. A. Baum, A. J. 60, I 54, I955; paper no. 66. Am. Astron. Soc. Meeting, April 1955.

[18] W. Baum and M. Schwarzschild, A. J. 60, 247, 1955.

[19] M. Schwarzschild, A. J. 59, 273, I954.

[20] W. Baade, Ap. J. 100, 137, r944.

[2I] W. Morgan and N. Mayall, P. A. S. P. 69, 29I, 1957.

[22] J. Stebbins and A. Whitford, Ap. J. 102, 334, I945; Ap. J. 108, 4I5, I948; M. N. 110, 42I, I950.

[23] M. S. Roberts, A. J. 61, 195, 1956.

[24] A. E. Whitford, A. J. 61, 352, 1956.

F. Zwicky, Morphological Astronomy p. 219, Springer, 1957.

[25] W. A. Baum, A. J. 62, 6, I957.

[26] W. G. Tifft, Ph. D. Thesis, Calif. Inst. of Tech., I958.

[27] W. A. Baum, P. A. S. P. 71, 106, I959.

[28] A. D. Code, private communication.

[29] W. Baade and H. Swope, Carnegie Inst. of Wash. Year Book 1956-7, p. $5^{8 .}$

[30] E. Holmberg, Medd. Lunds Astron. Obs., Ser. II, Nr. 136, $195^{8 .}$

[3I] M. Schwarzschild, Structure and Evolution of the Stars, p. 21 (Princeton University Press, 1958).

[32] E. M. Burbidge, G. R. Burbidge, W. A. Fowler, F. Hoyle, Rev. Mod. Phys., 29, 547, 1957. W. A. Fowler, J. L. Greenstein, Proc. N. A. S., 42, I73, 1956. 